TITLE:

\title{
DrugE-Rank: Predicting Drug-Target Interactions by Learning to Rank
}

$\operatorname{AUTHOR}(S)$ :

Deng, Jieyao; Yuan, Qingjun; Mamitsuka, Hiroshi; Zhu, Shanfeng

CITATION:

Deng, Jieyao ...[et al]. DrugE-Rank: Predicting Drug-Target Interactions by Learning to Rank. Methods in Molecular Biology 2018, 1807: 195-202

ISSUE DATE:

2018-07-21

URL:

http://hdl.handle.net/2433/236181

\section{RIGHT:}

This is a post-peer-review, pre-copyedit version of an article published in Methods in Molecular Biology. The final authenticated version is available online at: http://dx.doi.org/10.1007/978-1-4939-8561-6_14.; The full-text file will be made open to the public on 21 July 2019 in accordance with publisher's 'Terms and Conditions for Self-Archiving'.: This is not the published version. Please cite only the published version.; この論文は出版社版でありません。引用の際には 出版社版をご確認ご利用ください。 


\section{DrugE-Rank: predicting drug-target interactions by Learning to Rank}

Jieyao Deng ${ }^{1,2}$, Qingjun Yuan ${ }^{1,2}$, Hiroshi Mamitsuka ${ }^{3,4}$, Shanfeng Zhu ${ }^{1,2,5,{ }^{*}}$

${ }^{1}$ School of Computer Science and ${ }^{2}$ Shanghai Key Lab of Intelligent Information Processing, Fudan University, Shanghai, China,

${ }^{3}$ Bioinformatics Center, Institute for Chemical Research, Kyoto University, Uji 6110011, Japan, ${ }^{4}$ Department of Computer Science, Aalto University, 02150, Espoo, Finland and ${ }^{5}$ Center for Computational System Biology, Fudan University, Shanghai 200433, China

Email: zhusf@fudan.edu.cn;

* Corresponding author 
Deng et al.

\title{
DrugE-Rank: Drug-target Interaction Prediction by Learning to Rank
}

Jieyao Deng ${ }^{1,2}$, Qingjun Yuan ${ }^{1,2}$, Hiroshi Mamitsuka ${ }^{3,4}$, Shanfeng Zhu ${ }^{1,2,5, *}$

${ }^{1}$ School of Computer Science and ${ }^{2}$ Shanghai Key Lab of Intelligent Information Processing, Fudan University, Shanghai, China, ${ }^{3}$ Bioinformatics Center, Institute for Chemical Research, Kyoto University, Uji 6110011, Japan, ${ }^{4}$ Department of Computer Science, Aalto University, Espoo 02150 Finland, and ${ }^{5}$ Center for Computational System Biology, Fudan University, Shanghai 200433, China

\begin{abstract}
Identifying drug-target interactions is crucial for the success of drug discovery. Approaches based on machine learning for this problem can be divided into two types: feature-based and similarity-based methods. By utilizing the 'Learning to rank' framework, we propose a new method, DrugE-Rank, to combine these two different types of methods for improving the prediction performance of new candidate drugs and targets. DrugE-Rank is available at http://dataminingiip.fudan.edu.cn/service/DrugE-Rank/.
\end{abstract}

Keywords: DrugE-Rank, learning to rank, drug discovery, 


\section{Introduction}

Identifying drug-target interactions is crucial for the success of drug discovery. It can facilitate the understanding of drug side effect [1-3], disease pathology, as well as the drug action mechanism. Compared with using biochemical experiments to identify drug target interaction, computational approaches are more efficient and economical. Approaches based on machine learning for this problem can be divided into two types: feature-based and similarity-based methods [4-6]. By utilizing the 'Learning to rank' (LTR) [7-8] framework, we propose a new method, DrugE-Rank [9], to combine these two different types of methods for improving the prediction performance of new candidate drugs and targets.

We are interested in the problem of predicting drug-target interactions for new drugs or new targets. This problem is especially challenging, due to three main reasons. Firstly, since there are no known interactions for new drug or target, the training of prediction models is difficult. Secondly, existing computational methods based on LTR do not consider the connections among different drugs or targets very well. Thirdly, the prediction of drug target interaction is a challenging multilabel learning problem, where a new target (or drug) has multiple interacting drugs (or targets).

Compared with previous computational approaches, DrugE-Rank has multiple advantages. Firstly, by utilizing the LTR paradigm, DrugE-Rank can solve this multi-label learning problem naturally and provide the most powerful performance. Secondly, DrugE-Rank integrates diverse cutting-edge techniques in the framework of LTR, which include both similarity-based and feature-based methods. Thirdly, DrugE-Rank only considers the top drug (or target) candidates recommended by each component method, which can greatly reduce the computational burden. 
Deng et al.

\section{Materials}

The performance of DrugE-Rank was examined by using DrugBank [10], a manually annotated drug target interactions database. We carried out three rounds of experiments. I) cross validation over DrugBank data with FDA approved drugs before March 2014; 2) Independent test over DrugBank data with new targets and FDA approved drugs after March 2014; 3) Independent test over FDA experimental drugs. The experimental results demonstrate that DrugE-Rank outperformed all competing methods, being statistically significant. The improvement is especially promising for new drugs. Finally, we train DrugE-Rank with DrugBank data by the end of 2015. It consists of 1324 human protein targets, 1242 FDA approved drugs, and altogether 5484 known interactions.

\section{Methods}

Six cutting-edge similarity-based methods are used in DrugE-Rank as component methods in the LTR framework: Bipartite Local Model with support vector classification (BLM-Svc) [11], Bipartite Local Model with support vector regression (BLMsvr), k-Nearest Neighbor (k-NN) [12], Weighted Nearest Neighbor-based Gaussian Interaction Profile classifier (WNN-GIP) [13], Laplacian regularized least squares (LapRLS) [14], Network-based Laplacian regularized least squares(NetLapRLS). In addition, we extract drug features using RDKit (see Note 1), and target features from PROFEAT [15].

\section{Usage}

\subsection{New Drug}

Given a new drug, DrugE-Rank returns the top 20 targets as the predicted result. The input interface is shown in Figure 1.

1. Choose input format. You can input the drug profile by DrugBank ID, SMILES or MOL Format Text. An example of input is shown in Figure 2. 
2. Click the "Send" button. Click the button at the bottom of the page and your task will be in processing. The process takes about 10 minutes, and the server will return the top 20 predictions for each method (DrugE-Rank and six similarity-based methods). The result can help you to prioritize the most promising targets (Figure 3).

\subsection{New target}

Given a new target, DrugE-Rank returns the top 20 drugs as the predicted result. The input interface is shown in Figure 4.

1. Choose input format. You can input the target profile by UniProt ID or Amino Acid Sequence (Fasta format). An example of input is shown in Figure 5.

2. Click the "Send" button. Click the button at the bottom of the page and your task will be in processing. The process takes around 10 minutes, and the server will return the top 20 predictions for each method (DrugE-Rank and six similarity-based methods). The result may help you to prioritize the most promising drugs (Figure 6).

\section{Notes}

\section{1. http://www.rdkit.org/}

\section{Acknowledgements}

This work has been partially supported by National Natural Science Foundation of China (Grant Nos: 61572139), MEXT KAKENHI \#16H02868 and FiDiPro by Tekes. 
Deng et al.

\section{References}

[1] Keiser MJ, Setola V, Irwin JJ et al. (2009). Predicting new molecular targets for known drugs. Nature, 462(7270), 175-181.

[2] Lounkine E, Keiser MJ, Whitebread S, et al. (2012). Large-scale prediction and testing of drug activity on side-effect targets. Nature, 486(7403), 361-367

[3] Nunez S, Venhorst J, Kruse CG. (2012). Target-drug interactions: first principles and their application to drug discovery. Drug Discov Today, 17, 10-22

[4] Ding H, Takigawa I, Mamitsuka H, Zhu S. (2014). Similarity-based machine learning methods for predicting drug-target interactions: a brief review. Briefings in Bioinformatics, 15(5), 734-747.

[5] Zheng X, Ding H, Mamitsuka H, Zhu S. (2013). Collaborative matrix factorization with multiple similarities for predicting drug-target interactions. In Proceedings of the 19th ACM SIGKDD international conference on Knowledge discovery and data mining, pages 1025-1033. ACM.

[6] Takigawa, I and Mamitsuka, H. (2013). Graph Mining: Procedure, Application to Drug Discovery and Recent Advance. Drug Discov Today, 18 (1-2), 50-57

[7] Liu T. (2009). Learning to rank for information retrieval. Foundations and Trends in Information Retrieval, 3(3), 225-331.

[8] Li H. (2011). A short introduction to learning to rank. IEICE Transactions, 94-D(10), 18541862.

[9] Yuan Q, Gao J, Wu D, et al. DrugE-Rank: improving drug-target interaction prediction of new candidate drugs or targets by ensemble learning to rank. Bioinformatics, 2016, 32(12):i18-i27.

[10]Law V, Knox C, Djoumbou Y, Jewison T, Guo AC, Liu Y, Maciejewski, A., Arndt D, Wilson M, Neveu V, et al. (2014). Drugbank 4.0: shedding new light on drug metabolism. Nucleic acids research, 42(D1), D1091-D1097.

[11]Bleakley K, Yamanishi Y. (2009). Supervised prediction of drug-target interactions using bipartite local models. Bioinformatics, 25(18), 2397-2403.

[12]Van LT, Marchiori E. (2013). Predicting drug-target interactions for new drug compounds using a weighted nearest neighbor profile. PloS one, 8(6), e66952.

[13]Van LT, Nabuurs SB, Marchiori E. (2011). Gaussian interaction profile kernels for predicting drug-target interaction. Bioinformatics, 27(21), 3036-3043.

[14]Xia Z, Zhou X, Sun Y, Wu L. (2009). Semi-supervised drug-protein interaction prediction from heterogeneous spaces. In The Third International Symposium on Optimization and Systems Biology, volume 11, pages 123-131.

[15]Rao H, Zhu F, Yang G, Li Z, Chen Y.(2011). Update of profeat: a web server for computing structural and physicochemical features of proteins and peptides from amino acid sequence. Nucleic acids research, 39(suppl 2), W385-W390. 


\section{DrugE-Rank New Drug New Target Information Experiments-}

\section{Input Drug/Compound Profile}

DrugBank ID (e.g. DB00117):

or SMILES (Simplified Molecular Input Line Entry System):

or MOL Format Text:

\section{SEND}

Copyright (C) 2016 Shanghai Key Lab of Intelligent Information Processing and School of Computer Science, Fudan University, Shanghai 200433, China. All Rights Reserved.

Figure 1: Input interface for New Drug 
Deng et al.

\section{DrugE-Rank New Drug New Target Information Experiments-}

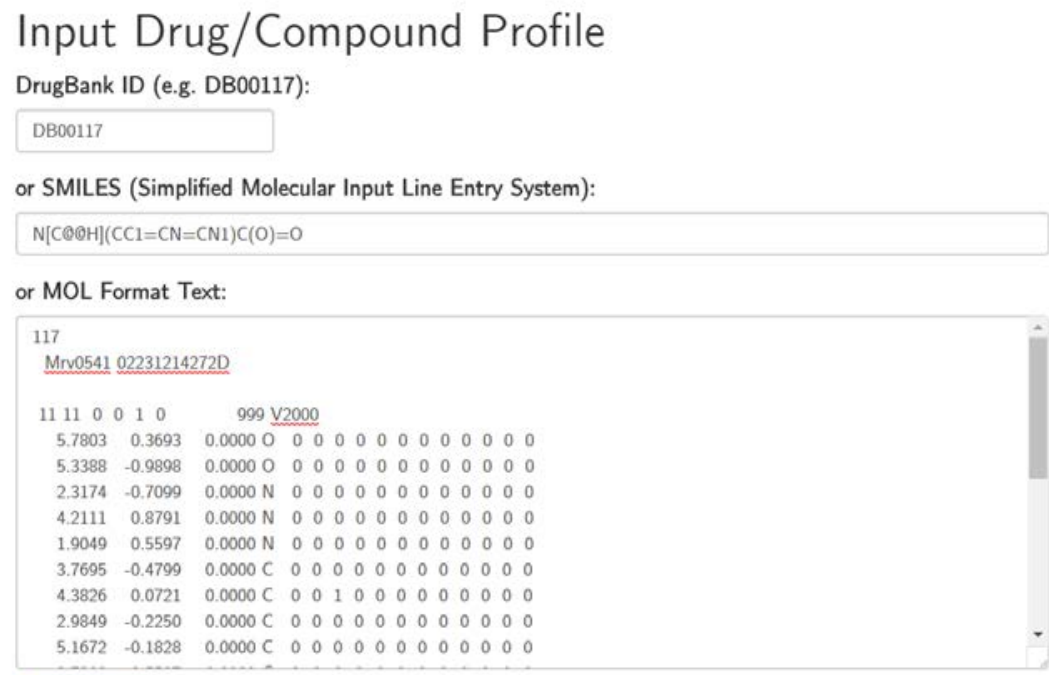

\section{SEND}

Copyright (C) 2016 Shanghai Key Lab of Intelligent Information Processing and School of Computer Science, Fudan University, Shanghai 200433, China. All Rights Reserved.

Figure 2: Input example for New Drug 


\section{Input Drug/Compound Profile}

DrugBank ID (e.g. DB00117):

DB00117

or SMILES (Simplified Molecular Input Line Entry System):

or MOL Format Text:

\section{SEND}

\section{Predicting Result (Top 20 predictions for each method)}

\begin{tabular}{|c|c|c|c|c|c|c|c|}
\hline$\#$ & DrugE-Rank & kNN & BLM-sve & BLM-svr & LapRLS & NetLapRLS & WNN-GIP \\
\hline 1 & P19113 & Q99624 & Q99624 & Q99624 & Q99624 & Q99624 & Q99624 \\
\hline 2 & P42357 & P19113 & P12081 & P19113 & P19113 & P19113 & P12081 \\
\hline 3 & P12081 & P42357 & P19113 & P42357 & P42357 & P42357 & P42357 \\
\hline 4 & Q99624 & P12081 & P42357 & P12081 & P12081 & P12081 & P19113 \\
\hline 6 & P08172 & P30825 & P08172 & P17812 & P08172 & P08172 & P29474 \\
\hline 7 & P11229 & Q8WY07 & P35348 & P35218 & P11229 & P11229 & P20309 \\
\hline 8 & 043246 & P78540 & P20309 & P00734 & P18089 & P29474 & P35228 \\
\hline 9 & P29474 & P08243 & P08913 & P37288 & P08913 & P35228 & O43246 \\
\hline 13 & P35228 & P21917 & P08908 & 000329 & P29474 & Q8WY07 & P78540 \\
\hline 14 & P08913 & P21728 & P21728 & Q13370 & P08908 & P00966 & P11229 \\
\hline 15 & P18089 & P35462 & P08173 & Q9HCR9 & P35462 & Q96A70 & P04424 \\
\hline 16 & P23219 & P14416 & P08912 & P42338 & P21728 & P04424 & Q16850 \\
\hline 17 & P18825 & P23219 & P35368 & P42336 & P41595 & P28222 & P23219 \\
\hline 18 & P21728 & P08913 & P28335 & Q14643 & P21917 & P23219 & P08243 \\
\hline 19 & P20711 & P18089 & P28223 & Q13315 & P35228 & P32297 & Q96A70 \\
\hline
\end{tabular}

Figure 3: Output example for New Drug 
Deng et al.

\section{DrugE-Rank New Drug New Target Information Experiments-}

\section{Input Target/Protein Profile}

UniProt ID (e.g. P19113):

or Amino Acid Sequence (can be in FASTA format):

\section{SEND}

Copyright (c) 2016 Shanghai Key Lab of Intelligent Information Processing and School of Computer Science, Fudan University, Shanghai 200433, China. All Rights Reserved.

Figure 4: Input interface for New Target 
DrugE-Rank New Drug New Target

\section{Input Target/Protein Profile}

UniProt ID (e.g. P19113);

P19113

or Amino Acid Sequence (can be in FASTA format):

$>$ sp |P19113|DCHS HUMAN Histidine decarboxylase $\mathrm{OS}=$ Homo sapiens $G N=H D C P E=1 \quad \mathrm{SV}=2$

MMEPEEYRERGREMVDYICQYISTVRERRVTTPDVQPGYLRAQLPESAPEDPDSSWDSIFGD

IERIIMPGVVHWOSPHMHAYYPALTSWPSLLGDMLADAINCL GETWASSPACT ELEMNVM

DWLAKMLGLPEHFLHHHPSSQGGGVLQSTVSESTLLALLAARKNKILEMKTSEPDADESC

LNARLVAYASDQAHSSVEKAGLISLVKMKFLPVDDNFSLRGEALQKAIEEDKQRGLVPVF

VCATLGTTGVCAFDCLSELGPICAREGLWLHIDAAYAGTAFLCPEFRGFLKGIEYADSFT

FNPSKWMMVHFDCTGFWVKDKYKLQQTFSVNPIYLRHANSGVATDFMHWQIPLSRRFRSV

KLWFVIRSFGVKNLQAHVRHGTEMAKYFESLVRNDPSFEIPAKRHLGGVVFRLKGPNCLT

ENVLKEIAKAGRLFLIPATIQDKLIIRFTVTSOFTTRDDILRDWNLIRDAATLILSOHCT

SQPSPRVGNLLISQIRGARAWACGTSLQSVSGAGDDPVQARKIIKQPQRVGAGPMKRENGL

HLETLLDPVDDCFSEEAPDATKHKLSSFLFSYLSVQTKKKTVRSLSCNSVPVSAQKPLPT

EASVKNGGSSRRVRIFSRFPEDMMMLKKSAFKKLIKFYSVYPSFPECSSQCGLQLPCCPPLQA

MV

\section{SEND}

Copyright (c) 2016 Shanghai Key Lab of Intelligent Information Processing and School of Computer Science, Fudan University, Shanghai 200433, China. All Rights Reserved.

Figure 5: Input example for New Target 
Deng et al.

\author{
Input Target/Protein Profile \\ UniProt ID (e.g. P19113): \\ P19113 \\ or Amino Acid Sequence (can be in FASTA format):
}

\title{
SEND
}

\section{Predicting Result (Top 20 predictions for each method)}

\begin{tabular}{|c|c|c|c|c|c|c|c|}
\hline \# & DrugE-Rank & kNN & BLM-svc & BLM-svr & LapRLS & NetLapRLS & WNN-GIP \\
\hline 1 & DB00142 & DB00117 & DB05266 & DB00117 & DB00157 & DB00157 & DB01235 \\
\hline 2 & DB00157 & DB00968 & DB05260 & DB00619 & DB00142 & DB00142 & DB00765 \\
\hline 3 & DB00898 & DB00190 & DB00830 & DB00193 & DB00898 & DB00898 & DB00667 \\
\hline 4 & DB00201 & DB00142 & DB00843 & DB01034 & DB00334 & DB00334 & DB01085 \\
\hline 5 & DB00117 & DB00151 & DB00849 & DB00201 & DB00201 & DB00201 & DB00782 \\
\hline 6 & DB00334 & DB00780 & DB00847 & DB01219 & DB01049 & DB01049 & DB00997 \\
\hline 7 & DB01049 & DB00149 & DB06795 & DB01043 & DB00171 & DB00171 & DB00376 \\
\hline 8 & DB00171 & DB00160 & DB06335 & DB00202 & DB00143 & DB00143 & DB00416 \\
\hline 9 & DB00143 & DB00242 & DB06589 & DB00606 & DB00543 & DB00543 & DB06262 \\
\hline 10 & DB00543 & DB00157 & DB00411 & DB00869 & DB00909 & DB00909 & DB00221 \\
\hline 11 & DB00421 & DB00116 & DB00653 & DB00880 & DB00321 & DB00321 & DB00174 \\
\hline 12 & DB00909 & DB00653 & DB00412 & DB01194 & DB00139 & DB00139 & DB00181 \\
\hline 13 & DB05266 & DB00661 & DB00651 & DB00626 & DB00421 & DB00421 & DB01253 \\
\hline 14 & DB00321 & DB00421 & DB00894 & DB00875 & DB00786 & DB00786 & DB00723 \\
\hline 15 & DB01235 & DB00622 & DB00656 & DB05246 & DB00408 & DB00408 & DB00988 \\
\hline 16 & DB05260 & DB00401 & DB00419 & DB00347 & DB01159 & DB01159 & DB00191 \\
\hline 17 & DB00653 & DB04855 & DB00418 & DB00593 & DB00145 & DB00145 & DB00286 \\
\hline 18 & DB00619 & DB01115 & DB00661 & DB01196 & DB00514 & DB00514 & DB00157 \\
\hline 19 & DB00968 & DB00270 & DB00422 & DB00562 & DB00312 & DB00312 & DB01337 \\
\hline 20 & DB00765 & DB00381 & DB00668 & DB01624 & DB00128 & DB00128 & DB01043 \\
\hline
\end{tabular}

Figure 6: Output example for New Target 